\title{
Nutrition for pets: navigating uncharted waters
}

\section{Opinion}

As a Canadian veterinarian in private practice I am responsible for providing truthful and practical advice to pet owners and unlike other areas of medicine, nutrition is always a highly emotional topic. Shortly after I emigrated to North America I was struck by the notion that food can be not only harmful, but sometimes even dangerous. This is not as obvious as it might seem. Growing up in Russia and raised on my family's first-hand memories of the war and ensuing famines, I brought with me the simple if naive understanding that food is good, whereas the absence of food is bad because it means starvation. To waste and throw away food is blasphemy. But to say that certain foods are harmful and to avoid them-what happened to produce this sad state of affairs? A large part of my work is devoted to undoing the damage done by food that is either inappropriate to the animal or fed inappropriately. Cats are a microcosm for this problem since of all veterinary species they are the hardest hit by the controversies and changing opinions in pet nutrition, whereas type II diabetes in cats and in humans are similar enough to warrant closer attention to this little masterpiece of an animal.

Veterinarians can be just as emotional about food as the pet owners they work with and educate. Gone are the days when a cat or dog could be maintained on table food, but there is no agreement in the profession on how to use science as a foundation for nutrition. There is among North American veterinarians an unspoken yet strong loyalty to major pet food companies (the "big four") based on the fact that these companies do extensive research that began in the 1950s with the first Hill's diet for Buddy, a German shepherd with ailing kidneys. As scientists we have innate respect for the concept of research, but such respect should not translate into religious acceptance of the bags and cans of food that emerge from a company's plant.

One problem is that research into animal nutrition has become part of mainstream science which in turn is governed by economics and the realities of agriculture. The human food pyramid is a good illustration of this principle: the starting point is a food source that is plentiful and relatively cheap to produce (wheat and corn), and the goal is to study how this source can be utilized in a way acceptable to health. The same approach is used in research into animal nutrition. However, acceptable is by far not the same as optimal. Cats are indeed capable of utilizing carbohydrates sourced from grains-by storing them away as body fat. The unfortunate popularity of dry foods is responsible for the epidemic of obesity among cats, for the common problem of urinary crystals that often lead to painful and life-threatening urinary obstruction in younger cats, and quite possibly for an earlier onset of kidney insufficiency in older cats who live in a state of sub-clinical dehydration if they do not drink enough water. (Descended from desert animals, cats do not have a thirst drive separate from hunger, as such do not have a reason to drink water.) The other problem is that of implementation. If a pet food company's research shows that rice has the highest glycemic index of commonly used carbohydrate sources, and that same company uses rice in a food designed for diabetic cats, it is wise to learn the scientific lesson but look for a food that reflects it better.

The public's disenchantment with major pet food companies has

\author{
Volume 2 Issue 5 - 2015
}

\author{
Veronica Gventsadze \\ Locum veterinarian, NA
}

Correspondence: Veronica Gventsadze, Locum veterinarian, \#204 40160Willow Crescent Squamish, British Columbia,V8B 0M2, NA, Tel 604849 1620, Email gassendus@hotmail.com

Received: January 13, 2015 | Published: September 21, 2015

led to a backlash against science itself and nostalgia for the poorly defined concept of what is "natural." It is easy to forget that the things we value about food and the experience of eating are very far from "natural." It is unnatural for animals (and people) to be picky about their food. It is unnatural and to a large extent unhealthy to be full and absolutely hunger-free all the time, yet freedom from hunger (itself a very subjective notion) is a basic requirement of modern life. It is unnatural for food to be addictively tasty, yet when dry kibble is coated with animal digest to enhance its flavor, a cat simply does not know when to stop eating. Finally, the very fact of our pets' longevity is unnatural, so we do not know with any amount of certainty what is optimal nutrition for a 10year-old dog or a 16year-old cat. We must embrace this unnaturalness as a small victory over entropy and learn to find our bearings in this new reality.

As veterinarians we need have only one loyalty: not to a particular pet food company or brand or even the idea of "natural," but to the health of the pet in front of us. When a client says they are proud to feed this or that food because it is grain-free and contains no gluten or animal by-products, it is our duty to explain why each of these points is important, or irrelevant, as the case may be. Cats simply do not need grains, and avoiding them also avoids the risk from aflatoxins associated with grains. Cats are unlikely to be harmed by gluten, which, contrary to popular opinion, is not a common allergen, and is a fairly good source of protein. As for animal by-products, a mouse's intestines are a hunting cat's favourite place to start a meal! Raw diets, often dismissed as unsafe and unbalanced, can be beneficial to cats with certain allergic conditions when properly prepared and supplemented. A common problem with commercially prepared raw foods is that owners often do not realize that the food is dehydrated and does not contain sufficient water to meet a cat's needs. In summary, we need to consider nutritional facts one at a time, and resist the temptation to make a discussion about food into an ideological debate.

As a truly international publication, the new Journal of Dairy, Veterinary and Animal Research is in a unique position to tap into the wisdom and experience of veterinary colleagues worldwide. This is especially true in the field of nutrition where North America may be a leader in research but has much to learn from the rest of the world about a healthy lifestyle for its pets and the humans who care for them and whose lives they brighten. 


\section{Acknowledgements}

None.

\section{Conflict of interest}

Author declares that there is no conflict of interest. 